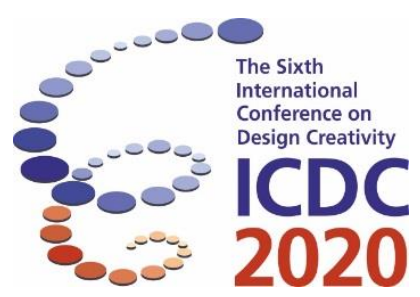

\title{
Process for Mapping Challenges of Cross-Border Mobility in the Barents Region
}

\author{
Mari Suoheimo $^{1}$ and Toni Lusikka ${ }^{2}$ \\ ${ }^{1}$ Faculty of Art and Design, University of Lapland, Rovaniemi, Finland \\ ${ }^{2}$ Transport and mobility, VTT Technical Research Centre of Finland Ltd, Oulu, Finland
}

\begin{abstract}
There is a challenge in identifying problems in common to tackle them creatively and jointly in Mobility as a Service (MaaS) development. This all requires collaboration and commitment, which is a wicked problem and a novel perspective in transportation and MaaS development. Mess $\mathrm{Map}^{\mathrm{TM}}$ is a tool to aid in creating shared understanding in cross-border mobility in the Barents region. Mess Map ${ }^{\mathrm{TM}}$ is a giant map that aims to map the whole complexity through a dialogue among the relevant stakeholders involved. In this case study, there were five transportation and MaaS projects besides other relevant stakeholders involved. The meetings were facilitated and run from a service design perspective that has a co-creational and holistic aim. The objective was to understand how the tool can be used in a service design process and how it can help the stakeholders to engage and find common goals.
\end{abstract}

Keywords: Cross-border mobility, complex stakeholder management, Mess Mapping ${ }^{\mathrm{TM}}$, service design, wicked problems

\section{Novelty of wicked problems in MaaS planning perspective}

Using the wicked problem perspective in transportation and mobility planning is something novel and we argue that it could be an important theory that could provide more effective groundbreaking results. Breaking silos and creating commitment and collaboration between different stakeholders is a major challenge (Eckhardt et al., 2018) if not even a wicked problem. Making a search at Scopus in November 2019, the search engine found 27 documents in total with the words "wicked problem* AND transportation OR mobility OR maas". More has been published on the topic only in the recent years 2014-2018. From these results, we think we can claim the perspective of wicked problems is novel in the field of MaaS planning and it could bring valid insights in the current discussions.

Wicked problem was a term and a theory coined by Rittel and Webber (1973). In total, 10 characteristics are required to make a problem a wicked one (Rittel \& Webber, 1973). We next intend to unfold these points from the perspective of transportation and MaaS development. It is hard to define what the actual problem in mobility planning is as it has many layers to be considered in designing them. Mobility planning has not got a stopping rule as it can always be improved, for example, with new technologies. It is hard to say that a mobility problem solution is "true-or-false", but rather better or worse. We use the word 'solution' still knowing that there are no optimal solutions to wicked problems. Making good solutions can improve the mobility and the subsistence of the people in the region, but bad ones can degrade the current status quo. For example, making a new road or railway across an indigenous land 
could bring severe unforeseen consequences to the local culture. The planners have not got the right to be wrong, because of those consequences. Planning each novel mobility service in its essence is unique. The mobility planning itself can be intertwined with many other wicked problems, such as unemployment, as many people depend on public transportation to go to work. It is challenging to make a formulation of the problem in the end, because how it is defined also influences how it will be treated. It is essential that the right stakeholders participate in the planning process and collaborative strategies have been set as an approach to treat wicked problems (Suoheimo, 2019; Suoheimo \& Miettinen, 2018;). In the transportation literature, we can find how researchers have defined the following themes as wicked problems: policy making of sustainable transportation (e.g. Givoni, 2013; Reardon \& Marsden, 2016; Ramani and Zietsman, 2016) governance in public transportation (Noto \& Bianchi, 2015); and making transportation analysis (Lyons, 2016). Lyons (2016) points out how stakeholder engagement is important in building better transportation futures. He also sees the value of multidisciplinary, if not even interdisciplinary work, in the wicked transportation context, which, in this sense, requires collaboration (Lyons, 2016).

Service design resonates with the afore mentioned issues. According to Stickdorn and Schneider (2011) service design is built upon five principles: 1) user-centeredness; 2) co-creation; 3) sequencing; 4) evidencing; and (5) holism. In the wicked problem setting, the designer or service designer has the role of a facilitator in an often complex stakeholder management context with collaborative approaches (e.g. Sepers, 2017; Prendiville, 2018; White and Koten, 2016). It is essential to bring holistic views to treat the issue from different perspectives (Sepers, 2017). The stakeholders present different areas and interests related to the problem and having them all involved, committed and collaborating can be a challenge. There has been a growing interest in the design and service design field regarding the tools that could be applied to wicked or ill-structured problems (e.g. Avdi et al., 2018; Suoheimo et al., 2020; Bofylatos \& Spyrou, 2016). Some tools have been specifically developed for the wicked problems perspective such as Mess Mapping ${ }^{\mathrm{TM}}$, Resolution Mapping ${ }^{\mathrm{TM}}$, General Morphological Analysis and Dialogue Mapping (Suoheimo, 2019). One thing in common for these tools is that they all value collaborative strategies by bringing stakeholders together to work from a holistic perspective (Suoheimo, 2019).

These tools could be a relevant alternative for the field to use to understand the complexities and interconnections. In mobility service planning, it is common to see tools such as SWOT-analysis; Value Network Analysis; initial reports often conducted by interviews; stakeholder analysis and stakeholder maps used to draw a picture of the problem field. As the transport sector can be considered a system-ofsystems, which consists of multiple sub-systems that can be categorized in different ways, it is necessary to use multi-disciplinary approaches to understand the system dynamics and behaviour (Leviäkangas, 2016). Hence, the Mess Map ${ }^{\mathrm{TM}}$ is more novel method for analysis than commonly used tools as the aforementioned ones. It could be a new way to gather multifaceted mobility issues in a holistic systemof-systems point of view, adding a new perspective to the current discussion on mobility planning. In the system-of-systems or in wicked problems, it is important to make limitations on what the subsystems or the problem areas are to be analysed, even though everything is connected.

We selected Mess Mapping ${ }^{\mathrm{TM}}$ as a tool to draw the complexity and to promote understanding about the messiness of cross-border mobility in the Barents region. As one of the participants in the kick-off meeting said, we should understand why the cross-border mobility is a mess. The map is made through workshops and is facilitated by a person that notes and draws down the participants' exchanges. Besides the common understanding of the problems, another challenge that the map aims to provide is a visualization of collaboration and commitment among the stakeholders. Making stakeholders come together in focus groups makes them interact and possibly create further collaboration and thus commitment to each other and to the common problems. With this initial perspective, our research questions are: 1) How did the Mess Map ${ }^{\text {TM }}$ help the projects and entities to identify common challenges in MaaS development? How did the Mess Map ${ }^{\mathrm{TM}}$ help to identify stakeholders for creating common strategy? 2) What are the advantages and disadvantages of using Mess Map ${ }^{\text {TM }}$ in service design projects?

For the first question, we did not wish to use the word 'problem', because you might not know exactly what the problem is, but you might have an overall image of the challenge. The first question has an 
under-question aimed to highlight the need to identify the stakeholders and the collaboration needed to create a common strategy. It is also vital to understand what the tool limitations are and how it could be improved in the context of service design lead facilitation. For this reason, we ask what the advantages and disadvantages of using the tool are. The contribution of this paper is to provide a novel perspective from the wicked problem theory and the use of Mess Map ${ }^{\mathrm{TM}}$ for people in mobility planning to start interacting from the very first stages of a project planning.

\section{Mess MappingTM}

Suoheimo and Miettinen (2018) discussed how complexity mapping and Mess Mapping ${ }^{\mathrm{TM}}$ could help develop commitment in planning in the mobility field. The Mess Mapping ${ }^{\mathrm{TM}}$ tool was developed by Horn and Weber (2007). It is a sequential tool; the map is made through focus groups and aims to aid the exchanges among the stakeholders involved (Horn, 2018). This does not mean that the stakeholders agree on the issue, but the different opinions can be gathered in the map. As Horn (2018) wrote, it is essential that the key people (also those with decision power) are involved so that the map has an effect. The way stakeholders define a wicked problem at hand also influences how the problem can be tamed. Mess Maps ${ }^{\mathrm{TM}}$ are normally created with a facilitator that gathers the conversations in the map parallelly when people discuss the topics. One challenge is that often people that come to Mess Map ${ }^{\mathrm{TM}}$ meetings already have a ready solution although they can see that the problem has interrelations with various problems (Horn, 2018). Horn (2018, p. 40) explains this well "They often attempt to start by "solving" the individual "problems" before there is a full enough understanding of how highly complex social messes are structured and of the forces and factors that have thus far prevented their resolution". Besides the interrelations of the problem areas on the map, there are also lines called causal links and their aim is to open the causes of the problems (Horn, 2018). Thus, people start to understand that simple solutions of one problem area should not be the target, but rather thinking of the complexity. The overall image of a Mess Map ${ }^{\mathrm{TM}}$ might look messy at first but everything is actually well labelled and those not previously involved can grasp the idea of what is happening. Horn (2018) recommends that the texts in the map should be understandable to anyone and the technical terms should be explained. Often people turnover is a problem in long projects or in governmental entities. The Mess Map ${ }^{\mathrm{TM}}$ can aid in this to update the newcomers and to show what was previously done (Horn, 2018).

\subsection{Process of Mess MappingTM Cross-Border Mobility}

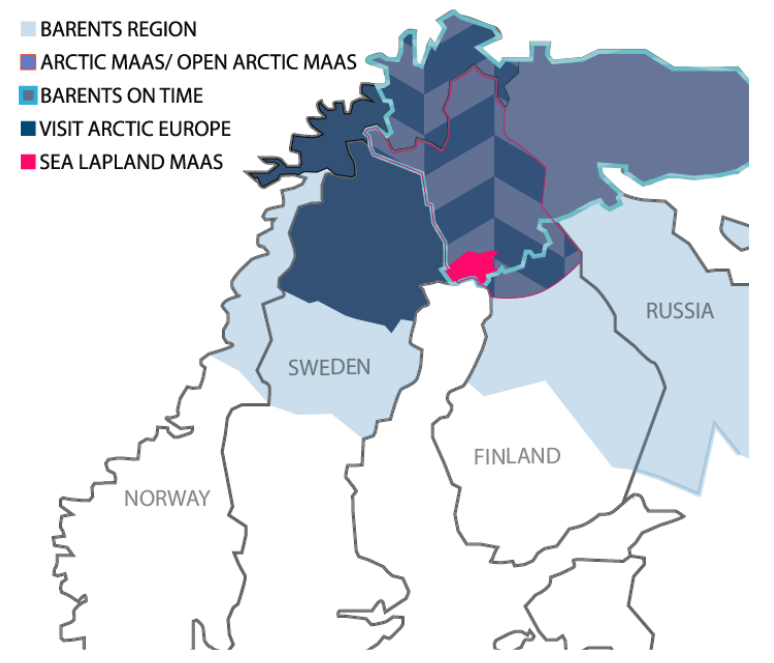

Figure 1. Projects involved and their geographical areas inside the Barents region.

This process of Mess Mapping ${ }^{\mathrm{TM}}$ is a single case study, whereby a total of five different mobility development projects in the Barents region were involved (Table 1). Figure 1 illustrates the geographical areas where the projects are located. Four of the five projects are Finnish led and one by Norway. We had focus group participants from Finland, Norway and Russia. The concentration was on cross-border 
mobility and, as one of the participants nicely expressed, it was good to come and collaborate as "we do the same things across the border".

Table 1. MaaS projects involved in the mapping.

\begin{tabular}{|c|c|c|c|c|}
\hline Project & 1) Barents on Time & 2-3) Open Arctic MaaS & $\begin{array}{l}\text { 4) Visit Arctic } \\
\text { Europe II }\end{array}$ & $\begin{array}{l}\text { 5) Sea Lapland } \\
\text { Tourism MaaS }\end{array}$ \\
\hline Website & $\frac{\text { https://kolarctic.info }}{\underline{I}}$ & https://cutt.ly/9t2q6ah & $\frac{\mathrm{https}: / / \text { cutt.ly/Zt2q }}{\underline{2 \mathrm{~h} 7}}$ & https://cutt.ly/8t2q92t \\
\hline Description & $\begin{array}{c}\text { Barents on Time is } \\
\text { a project that is } \\
\text { initiating } \\
\text { collaboration } \\
\text { between Finland, } \\
\text { Russia and Norway } \\
\text { and concentrates } \\
\text { mainly on cross- } \\
\text { border bus services. } \\
\text { It aims to make a } \\
\text { website and an app } \\
\text { to sell tickets for } \\
\text { cross-border } \\
\text { travellers. }\end{array}$ & $\begin{array}{l}\text { The Open Arctic MaaS } \\
\text { project portfolio consists of } \\
\text { two projects that have led to } \\
\text { the development of mobility } \\
\text { services in Northern Finland. } \\
\text { The projects have worked } \\
\text { towards promoting internal } \\
\text { accessibility and the } \\
\text { digitalisation of transport in } \\
\text { sparsely populated areas. } \\
\text { The vision is to significantly } \\
\text { promote and integrate local } \\
\text { and tourist mobility services } \\
\text { by making them easy and } \\
\text { reliable, thus enabling } \\
\text { sustainable travel. }\end{array}$ & $\begin{array}{l}\text { The Visit Arctic } \\
\text { Europe II project } \\
\text { concentrates on } \\
\text { developing year- } \\
\text { round, sustainable } \\
\text { and high-quality } \\
\text { tourism in Finnish } \\
\text { and Swedish } \\
\text { Lapland and in } \\
\text { Northern Norway. } \\
\text { Mobility planning } \\
\text { is essential in } \\
\text { order to bring the } \\
\text { tourists to their } \\
\text { destinations. }\end{array}$ & $\begin{array}{l}\text { In TourismMaaS, the } \\
\text { tourism product } \\
\text { itself is at the } \\
\text { forefront of the } \\
\text { service package } \\
\text { and the transfer is an } \\
\text { additional service. } \\
\text { Currently, } \\
\text { it is challenging to } \\
\text { link tourism and } \\
\text { mobility services. } \\
\text { The main goal of } \\
\text { the project is to link } \\
\text { the MaaS services as } \\
\text { part of the tourism } \\
\text { business. }\end{array}$ \\
\hline
\end{tabular}

The focus of the MaaS development in the Mess Map ${ }^{\mathrm{TM}}$ process was eventually more focused on bus transportation. In the initial discussions, there were plans also connected to ground transport and doorto-door services that it still broaches. The research process for mapping can be summarised in three phases: 1) Planning; 2) Co-discover phase, in which the map was made in collaboration with the different stakeholders; and 3) Co-define phase, in which evaluation and analysis were made.

\subsubsection{Planning}

The initial planning was made by the service design facilitator. Time was spent reading previous MaaS project reports and current projects applications and other relevant material found, such as scientific articles of the MaaS development in the region. On the basis of this knowledge, the first skeleton of the themes for Cross-Border Mobility Mess Map ${ }^{\mathrm{TM}}$ was made. Also, there were a couple of meetings and discussions with some stakeholders beforehand, listening to their needs and introducing the tool. A case study protocol (https://cutt.ly/FysSeWC) was made to ensure the validity of the study.

\subsubsection{Co-discover}

In total, there were 13 focus groups and the mapping process began with a kick-off meeting, when the participants together with a service design facilitator, defined what subproblems should be considered. Participants could make more proposals to the pre-suggested themes, such as the "Rescue planning". Of the 13 focus groups, 11 were held online (Business Skype) and two in person in Rovaniemi (Table 2). In total, there were 45 participants ( 20 different people) and around 19 hours of recordings. The whole process took about half a year. We decided not transcribe the audios because all the issues raised were immediately written down by the facilitator. The initial map was made in Google Drawings sheet so that everyone could have access to the map and also to write on it if needed. All the participants signed consent forms and the process follows the ethical guidelines of the European Union's Horizon 2020 framework programme. During the whole process, there were 13 participants from the projects, three 
from the users' perspective and six from other relevant entities. Different topics were discussed in each meeting. It was not necessary to have each person in every meeting as some of the topics were more specific, although they were always invited to all the projects, except the user experience meetings so that their identity would be preserved.

Table 2. Mess Map ${ }^{\mathrm{TM}}$ focus groups

\begin{tabular}{|c|c|c|c|c|}
\hline MEETINGS & Amount of focus groups & Place & Recording ap. & Participants \\
\hline TOTAL & 13 focus groups & 11 online; 2 in Rovaniemi & Ap. 19 hours & 45,20 different people \\
\hline
\end{tabular}

\subsubsection{Co-define, evaluation and analysis}

The data were evaluated through triangulation once multiple data collection methods were used to ensure the quality of the research (Bailey, 2017). The data were triangulated through mapping, evaluation forms and research diary with field notes. The writing process was made by two researchers and the data analysis was made in peer-review style meetings. In the final focus group, the map was analysed cocreatively, Figure 2. Participants were given three colours of pens so that each could point out challenges and causal links (black) to the problems; collaboration (green) and interrelations between the areas (red). These issues are important to visualize, because if one area is developed further, it will influence all the other interconnected areas. It was perceived that the red lines are connected to all the boxes and have two pointed arrows. Also, it was pointed out that more commitment and collaboration should occur practically between all of the boxes as the lines prove it and discussions were directed to it repeatedly. The focus group was conducted collaboratively and all the nine participants could express their points.

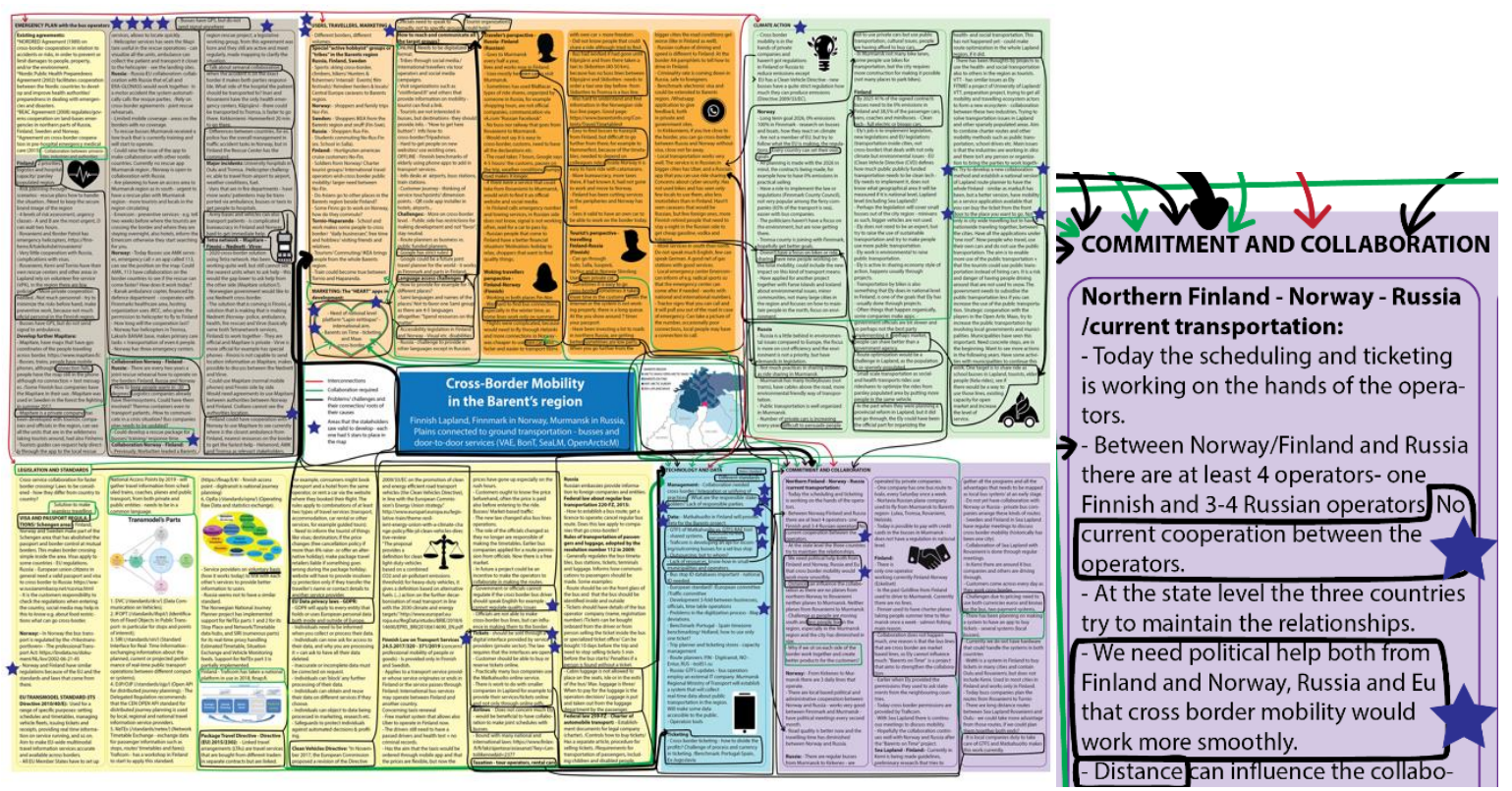

Figure 2. The final Mess Map ${ }^{\mathrm{TM}}$ and a detail of it. A link for an A1 size map: https://cutt.ly/mysStFg.

Only the ones attending the final meeting online or in person received the evaluation form as it contained questions treated in the last focus group. Seven out of the nine participants filled the evaluation form (Annex 1: https://cutt.ly/4ysSiP2). The evaluation form consisted of 11 open-ended questions and 11 statements that participants had to agree or disagree with by using a numerical scale from 5 to 1 . All the respondents had a university degree and the average time in the professional field was 12 years. The average age of the participants was ap. 41 years and the backgrounds were diverse, ranging from regional logistic, paramedic to university lecturer. None of the job titles were repeated, which shows the diversity. Most representatives were from public sector entities. Most were from Finland and only one from Russia, although there had been Norwegians and other Russians participating in earlier meetings. 
Unfortunately, some participants were unable to attend the final meeting. The tool and wicked problem perspective were explained in the three main meetings and some participants missed all of them and some heard two or three out of the three presentations.

First question regards how the Mess Map ${ }^{\mathrm{TM}}$ helped to identify common goals, strategy and the most important areas to develop. When analysing the responses, the participants' responses is observed to vary a bit and three themes arose. Two of them (FG01, FG02) elected collaboration as the most important area, while the rest of the participants chose technology and digitalization. Two participants (FG04, FG06) also felt that information about transport services must be made more available, which links their answers to digitalization. All the participants found common challenges with other projects and entities involved. From the answers, it can be seen that the common challenge is the collaboration with other stakeholders and projects both nationally and internationally. In addition, cultural and technological differences (e.g. standards) make the co-development process even more challenging.

An under-question of the first question concerns how the Mess Map ${ }^{\mathrm{TM}}$ helped to identify new stakeholders related to reaching the common strategy and goals. For some participants (FG01, FG04 and FG05), most of the other workshop-participants were familiar, but everyone found at least one part on the map that had new stakeholders to work with. New stakeholders were found both in the meetings and on the map. Also, some governmental stakeholders were deemed to be important to the projects, but had not previously met until the mapping. Two participants (FG01, FG04) felt that government officials, both national and international, should be more involved in finding a common strategy. Also, one participant (FG02) said that EU-level experts on transport legislation should be involved to find vast and long-term political will to co-create better mobility services. Participants (FG01, FG04) pointed out that entrepreneurs, such as bus companies, tourism companies, airlines and train companies, should be involved. All the participants think that they will continue working with other participants (and other stakeholders found on the map) towards common goals. Responses seem to be consistent with the main question: co-creation and collaboration with multiple stakeholders and projects is desired to form a united strategy and to have a clear political ambition towards better mobility services. Mess Map ${ }^{\mathrm{TM}}$ seems to have helped participants to identify the most important development areas and some of the participants also found new stakeholders to be involved in their development processes.

The second question regards the advantages and disadvantages of using the Mess Map ${ }^{\mathrm{TM}}$ tool. By analysing the open-ended responses from the evaluation form, one participant (FG01) felt that the tool was appropriate, pointing out the stakeholders and issues to go forward. Some said it gave much to think (FG04) or felt it took much time (FG02) or that it could be more condensed (FG05). Most reported that they felt that the problems were covered well and no-one said they would have more issues to raise. The improvements the participants provided for the focus groups were more in the technical issues, such as how to better take into consideration those that are online in a meeting when others are present (FG05, FG02); however, the people from the online side did not report it themselves and actually thought that the meetings worked well. One said (FG04) that the "paper", meaning the map, in the last meeting could have been larger although it was printed in an A1 size. There was a suggestion (FG02) to use the tool in other mobility issues with more specific scope.

By analysing the questions with a scale of one to five, the participants thought that by using the tool, we were able to map the problems of the cross-border mobility in the Barents region $(4,6)$. The participants thought that they were heard $(4,6)$ and that they learned something new during the process $(3,9)$ or had "new aha moments" (4). They also felt that their participation was relevant $(3,9)$ and that the voices of the users of the Barents region were heard $(3,9)$. Participants agree that they work with wicked problems $(4,4)$, but not so many thought that they will use the Mess Map ${ }^{\text {TM }}$ tool in the future (3), but saw that the tool was fit for their project or entity $(4,1)$. The first author of the article also kept a personal journal with field notes. The notes contain interesting aspects, such as one participant after a meeting commenting that he had found the day's focus group helpful to process their project and his thoughts around it. 


\section{Discussion and Conclusions}

The map was able to congregate quite a large number of people from different professional backgrounds to discuss the topics of cross-border mobility in the Barents region. To conclude the first question and its under-question, Mess Map ${ }^{\mathrm{TM}}$ can be used to identify common goals, strategy, targets for development, and relevant stakeholders. In addition, the mapping process helps participants to find and reach out to other stakeholders that are relevant for their mobility planning and development processes. The map can be used to show and explain the causalities, and thus the interconnectedness of different issues and stakeholders beyond the familiar and obvious ones. The challenges identified during the process relate to collaboration and commitment, while dealing with technological issues. In MaaS development, the greatest issue currently seems to be making collaborative public-private-people partnerships to reach their full potential also by breaking silos. Stakeholders and projects are working together more and more, but they lack resources, tools and methods to realize all the possibilities that collaboration could offer. By the same token, there is often a lack of commitment to solve common challenges and interconnected wicked problems. In addition, some stakeholders (or the organizations they represent) might not see the value of collaboration to be enough to invest resources in it, but this is done as 'extra work' during normal duties and procedures. We consider our results to be consistent and reliable even though the participant pool was quite limited in the final workshop. Also, as we are trying to understand mobility as a system-of-systems, it must be noted that the analysis of the system is limited by scoping the system itself and problem areas, methods applied to the analysis, and availability of data (Leviäkangas, 2016). In this mapping process, our scope was quite large; we were thus able to gather the majority of issues (that participants could perceive) related to cross-border mobility. Hence, the Mess Mapping ${ }^{\mathrm{TM}}$ process was very successful and could also be used in other contexts.

There are advantages and disadvantages over using the tool in the service design context facilitation. One disadvantage was that it was hard to make the MaaS users of the Barents region to participate or make their voices better heard. This was not considered relevant in one of the meetings by the participants although some deemed it necessary and extremely valid. We thus recommend using the Mess Mapping ${ }^{\mathrm{TM}}$ tool in combination with service design empathy building tools, such as service blueprints, where the actual planners would make some journeys and see what happens in the field as users. The advantage of using the Mess Mapping ${ }^{\mathrm{TM}}$ in the service design context is that there was a large number of stakeholders and their views were heard. Holistic perspective and collaboration, important to service design, was mapped. Some participants attended several meetings in full and others did not attend that many or did, but did not stay long. The responses from the latter group were more confusing, maybe because they did not have a general picture of the tool and its purpose for their lack of participation. Personally, as the facilitator, it was challenging to get the right people involved. Sometimes the people themselves did not understand that they were important to the topic until they came to the meeting. This could show how interconnected the problem is and how being a specialist in a certain area of the map makes the person important in a larger context. The largest number of participants were from Finland and we could therefore analyse the focus in greater depth from the Finnish perspective.

Based on the personal notes and peer-review meetings between the two authors, it seemed that some of the cultures of the participating countries did not support much the collaborative approach deriving from the principle that all are at a horizontal level. This participative approach is commonly practiced in Scandinavia and originates from the same geographical area (Sanoff, 2008; Simonsen \& Robertson, 2012), but may be very unfamiliar with the cultures without this tradition. Could this be the reason behind the underlying difficulty? We recommend future studies on how to use the tool in a context in which people are not that familiar with the collaborative view and find novel aspects for it. The Mess $\mathrm{Map}^{\mathrm{TM}}$ tool seems to be more of a novel compared to the many traditional ways of immersing to the MaaS challenges as it already starts with collaboration, making people come and meet each other from the very beginning. The process has been one way of creative collaborative thinking, in which the participants have been in the position of collaborating through their point of views and gaining insights from the mapping. More creativeness and innovations will be required when starting to tackle the issues often arising in situations that lack resources; this could also be a reason for collaboration. The scientific literature also shows how cross-disciplinary settings foster innovations. 


\section{Acknowledgements}

The first author would like to thank the Lapland Regional Fund of the Finnish Cultural Foundation for the oneyear grant for her $\mathrm{PhD}$ research. We also want to thank each of the MaaS project participants involved in the mapping process.

\section{References}

Avdiji, H., Elikan, D., Missonier, S., \& Pigneur, Y. (2018, January). Designing tools for collectively solving Illstructured problems. In Proceedings of the 51st Hawaii International Conference on System Sciences (pp. 400409).

Bailey, C. R., \& Bailey, C. A. (2017). A guide to qualitative field research. Sage Publications.

Bofylatos, S., \& Spyrou, T. (2016). Supporting design dialogue through a communication framework using four layers of abstraction. The Design Journal, 19(2), 269-282.

Eckhardt, J., Nykänen, L., Aapaoja, A., \& Niemi, P. (2018). MaaS in rural areas-case Finland. Research in Transportation Business \& Management, 27, 75-83.

Givoni, M. (2014). Addressing transport policy challenges through policy-packaging. 1-8.

Horn, R. E. (2018). The Little Book of Social Messes: Connecting the Smudges in an Age of Wicked Problems. Macro-Vu.

Horn, R. E., \& Weber, R. P. (2007). New tools for resolving wicked problems: Mess mapping and resolution mapping processes. Strategy Kinetics LLC.

Leviäkangas, P. (2016). Digitalisation of Finland's transport sector. Technology in Society, 47, 1-15. https://doi.org/10.1016/j.techsoc.2016.07.001.

Lyons, Glenn. (2016). Transport analysis in an uncertain world. Transport Reviews, 36(5), 553-557.

Noto, G., \& Bianchi, C. (2015). Dealing with multi-level governance and wicked problems in urban transportation systems: The case of Palermo municipality. Systems, 3(3), 62-80.

Prendiville, A. (2018). Amplifying Relationships through Place and Locality in the Design of Local Government Digital Services. She Ji: The Journal of Design, Economics, and Innov ation, 4(1), 47-59.

Ramani, T. L., \& Zietsman J. (2016). Sustainable transportation-alternative perspectives and enduring challenges. International Journal of Urban Sciences, 20(3), 318-333.

Reardon, L., \& Marsden, G. (2016). Steering Demand-A Wicked Problem in the Making: Insights from UK Transport Policy. Lancaster University.

Rittel, H. W., \& Webber, M. M. (1973). Dilemmas in a general theory of planning. Policy sciences, 4(2), $155-169$. Sanoff, H. (2008). Multiple views of participatory design. International Journal of Architectural Research: ArchNet-IJAR, 2(1), 57-69.

Stickdorn, M., \& Schneider, J. (2011). This is service design thinking: basics, tools, cases. Wiley.

Sepers, F. (2017). An Integral Design Framework-designing a global village. The Design Journal, 20(sup1), S1566-S1579.

Simonsen, J., \& Robertson, T. (Eds.). (2012). Routledge international handbook of participatory design. Routledge.

Suoheimo, M. (2019). Strategies and Visual Tools to Resolve Wicked Problems. The International Journal of Design Management and Professional Practice, 13 (2), 25-41, ISSN: 2325-162X.

Suoheimo, M., \& Miettinen, S. (2018). Complexity Mapping and Mess Mapping Tools for Decision-Making in Transportation and Maas. Proceedings of the 21st DMI: Academic Design Management Conference (pp. 11761188). London, Great Britain: Ravensbourne University.

Suoheimo, M., Vasques, R., \& Rytilahti, P. (2020). Deep Diving into Service Design Problems: Visualizing the Iceberg Model of Design Problems through a Literature Review on the Relation and Role of Service Design with Wicked Problems, The Design Journal, to be published.

White, R. M., \& van Koten, H. (2016). Co-designing for sustainability: strategizing community carbon emission reduction through socio-ecological innovation. The Design Journal 19(1): 25-46. 